\title{
AE Sound Source Localization Using Nearfield MUSIC Algorithm Based on Fourth-Order Cumulants
}

\author{
Jing $\mathrm{Li}^{1}$, Yong Yang ${ }^{2,4}$, Xinghua $\mathrm{Li}^{3}$ and $\mathrm{Li}$ Zhao ${ }^{1}$ \\ ${ }^{1}$ School of Information Engineering, Southeast University, Nanjing, China \\ ${ }^{2}$ School of Resources and Geosciences, China University of Mining and \\ Technology, Xuzhou, China \\ ${ }^{3}$ Chengdu BOE Optoelectronics Technology Co., Ltd., Chengdu, China \\ ${ }^{4}$ School of Information and Electrical Engineering, Xuzhou, China \\ 230149053@seu.edu.cn
}

\begin{abstract}
Acoustic Emission (AE) source localization is a useful implement to diagnose the incipient faults in rotating machine. This paper proposes an improved Near-field Multiple Signal Classification method using four-order cumulants (NFC-MUSIC) to accurately locate the faults position. In order to overcome dispersion effect and revised velocity, the feature sub narrow band is extracted by Wavelet Packet reconstitution (WPR) and Modal Plate Wave Theory (MPWT). For multi-source decorrelation and increasing localized resolution, the four-order cumulants of observed signal can be selected for localization. The experiment results indicate that the improved method can accurately locate multirubbing faults. It is an efficient way to assist incipient rubbing faults diagnosis.
\end{abstract}

Keywords: Rubbing Fault, Acoustic Emission, Near Field, Multiple Signal Classification

\section{Introduction}

The rotor elements are critical components of rotating machinery in industrial applications (i.e., turbines, compressors, pumps motors, etc.) [1].The scratching between rotor and stator emits acoustic energy which contains lots of fault information. Currently, acoustic emission (AE) technique served as a kind of rotor-stator diagnostics method can make full use of these information for the defects detection in its incipient stage and inform the operator before hazard occurring so as to keep the rotating machinery at healthy condition for maximum production.

Rubbing localization is of great importance in analysis of working condition of the machinery. As a conventional AE source localized method, Time difference of arrival algorithm (TDOA) has become the popular tool to recognize the AE source in laboratory. However, the localized accuracy of TODA is sensitive to preset AE signal threshold, dispersion and effective velocity during propagation process of waves and background noise etc., [2]. Besides, it is inconvenient and rather costly to use TDOA method for large rotary machinery since its large scale distribution of sensors for signal reception.

He [4] proposed another array signal processing method, Near field Beam forming method (N-BF) combined with the plate theory was particularly suited for the rotor-stator rubbing source localization than traditional methods due to the lower needs of high sampling rates, simplified sensor arrangement and avoiding dispersion effect. However, the localization accuracy is still threatened by the noise rooting in AE records. Afterward, in order to solve the $\mathrm{AE}$ essence properties problems such as: fast fading and the background noise Deng [5] investigated rubbing location algorithm of N-BF based on 
minimum variance distortionless response (MVDR). However, these improved methods can detect only one AE source at once under high signal-to-noise ratio (SNR).

Multiple signal classification (MUSIC) algorithm is an effective way to detect multiple acoustic sources simultaneously in far-field propagation model. Li applied MUSIC (NMUSIC) method into near field propagation model to estimate the localization of single $\mathrm{AE}$ source in rotor-stator rubbing using a uniform line array [6]. Tang presented an improved near field method (IN-MUSIC) to localize the two incoherent narrow band AE sources [7]. However, combined with the characteristics of AE source caused by rubimpact movement of rotor-stator such as low-strength, rapid attenuation, coherent and wideband, these nearfield MUSIC algorithm can hardly be suitable for rubbing fault localization.

This paper proposes a novel Near-field MUSIC algorithm for the improved localization accuracy of multiple rubbing faults in rolling element. The pre-processing using Wavelet Packet reconstitution (WPR) based on Modal Plate Wave Theory (MPWT) is utilized to extract the useful narrow band from the observed $\mathrm{AE}$ wideband signal and revise velocity. The fourth-order cumulants (FC-MUSIC) is applied to increase the aperture size in virtual way for the decorrelation and the higher resolution. The rest of this paper is organized as following. First, we briefly analysis MPWT for the pre-processing of AE signals in Section 2. Then the proposed model for AE source localization is presented in Section 3. Section 4 shows the experimental results in detail. Finally, we conclude this paper in Section 5 .

\section{The Pre-Processing of AE Signals.}

Based on the experiment condition the AE source can be transmitted in the waveguide steel plate with $10 \mathrm{~mm}$ thickness, which is much shorter than acoustic wavelength in accordance with MPWT assumption. Thus, the wave equations can be used to explain the propagation characteristics of AE signal [4]. Equation (1) and Equation (2) defined the relation between frequency and wave velocity of extensional wave(S) and flexural wave (A) respectively. Here the propagation velocity of longitudinal wave $c_{p}$ and the propagation velocity of shear wave $c_{\mathrm{s}}$ can be achived by using a lookup table method. Others $p$ and $q$ are two temporary variables, $k$ is the wave number, $\mathrm{h}$ is the half thickness of plate and $\omega$ is the circular frequency, the outputs $c$ is the phase velocity and $c_{g}$ is the group velocity,

$$
\begin{aligned}
& \frac{\tan (\mathrm{qh})}{\tan (\mathrm{ph})}=-\frac{4 k^{2} p q}{\left(q^{2}-k^{2}\right)^{2}} \\
& \frac{\tan (\mathrm{qh})}{\tan (\mathrm{ph})}=-\frac{\left(q^{2}-k^{2}\right)^{2}}{4 k^{2} p q} \\
& \mathrm{p}^{2}=\frac{\omega^{2}}{\mathrm{c}_{\mathrm{p}}^{2}}-k^{2}, \mathrm{q}^{2}=\frac{\omega^{2}}{\mathrm{c}_{\mathrm{s}}^{2}}-k^{2}, k=\frac{\omega}{\mathrm{c}}, \mathrm{c}_{g}=\frac{\partial \omega}{\partial k}
\end{aligned}
$$

As shown in Figure 1, the frequency is set below $200 \mathrm{MHz}$, the mode A0 and S0 can be reserved and the high order modes can be filtered away for decreasing the frequency dispersion effect. On the other hand, the previous research presented that all the generated rubbing $\mathrm{AE}$ frequency are approximately concentrated on the range from $20 \mathrm{kHz}$ to 50 $\mathrm{kHz}$ [5]. Thus, considering the integrity of the observed AE signal, the filter band of acquisition system should be set at 1-200 kHz. And further the major components should be extracted by multi-scale wavelet decomposition and reconstitution [9]. 

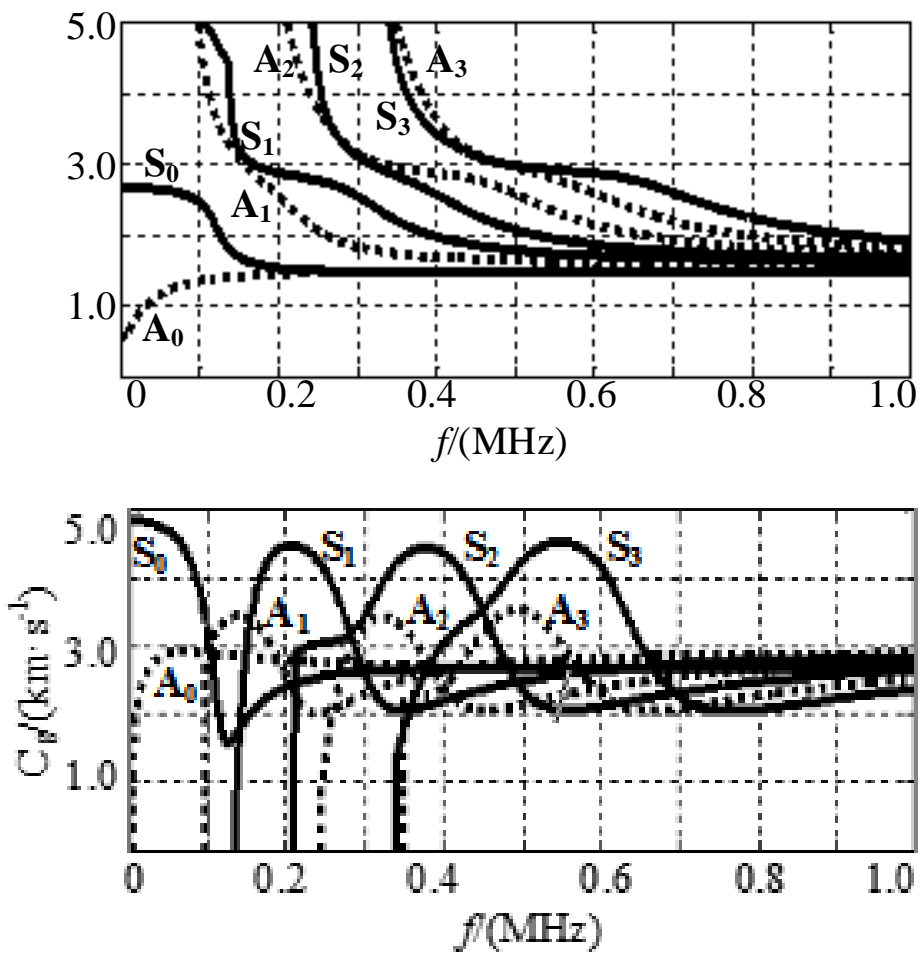

\section{Figure 1. Four Orders Dispersion Curves (a) Phase Velocity (b) Group Velocity}

Hemmati F concluded that the Daubechies11 (db11) orthogonal mother wavelet can achieved the optimal output SNR for the rubbing fault AE signal [8]. Then, the observed $\mathrm{AE}$ signal can be decomposed into three levels, the second sub-band with frequency range from 25.625 to $50.25 \mathrm{kHz}$ defined as the characteristic signal for the following localization.

\section{The Proposed AE Source Localization Algorithm.}

The region of near field source can be expressed as $|r| \leq 2 d^{2} / \lambda$, where $|r|$ is the distance form source to sensor, $d$ is the aperture array of linear array sensors and $\lambda$ is the operating wavelength. As shown in Figure 2, source 1 and source 2 are the preset coherent AE sources respectively. $\mathbf{r}_{p m}$ and $\boldsymbol{\theta}_{p m}$ are the distance and angle of arrival from the $p$-th AE source to the $m$-th sensor separately. The output of the sensors reception can be demonstrated by Equation (3), where $p(p<m)$ is the number of the sources, $\mathbf{x}_{\mathbf{m}}(\mathrm{t})$ is the observed records from the $m$-th sensor, $\tau_{i}$ is the arrival time difference between the $m$-th sensor and the reference sensor, $\mathrm{j}=\sqrt{(-1)}$ is the imaginary unit, $\mathbf{a}\left(r_{p}, \theta_{p}\right)$ is the steering vector of the $p$-th sources, $\mathbf{s}_{\mathbf{m}}(\mathrm{t})$ is the AE source signal and $\mathbf{e}_{\mathbf{m}}(\mathrm{t})$ is the noise vector. 


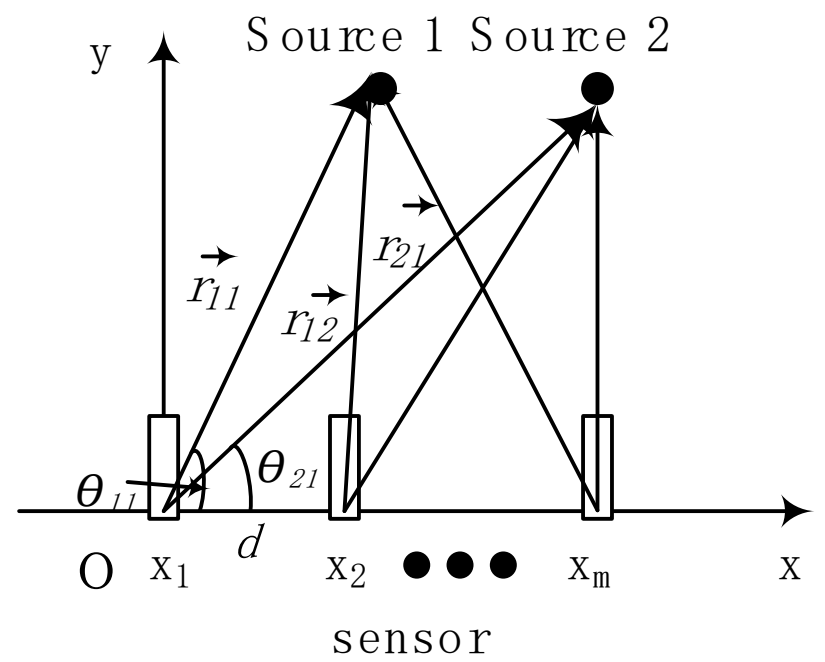

Figure 2. Illustration of Near-Field Localization Model

$\tau_{m}=\frac{\sqrt{r_{p 1}^{2}+(\mathrm{m}-1)^{2} d^{2}-2 r_{1}(\mathrm{~m}-1) d \cos \theta_{p 1}}-r_{p 1}}{c}$

$\mathbf{a}\left(r_{p}, \theta_{p}\right)=\exp \left[\mathrm{j} 2 \pi f \tau_{m}\right]$

$\mathbf{x}_{\mathbf{m}}(\mathrm{t})=\mathbf{a}\left(r_{p}, \theta_{p}\right) \mathbf{s}_{\mathbf{m}}(\mathrm{t})+\mathbf{e}_{\mathbf{m}}(\mathrm{t})$

Then, we adopt the fourth-order cumulants of the observed data for angle and distance of arrival. The definition of the fourthorder cumulants of $\mathrm{x}_{\mathrm{i}}(\mathrm{t}), \mathrm{x}_{j}^{*}(\mathrm{t}), \mathrm{x}_{k}(\mathrm{t})$ and $\mathrm{x}_{l}^{*}(\mathrm{t})$ is given by

$$
\begin{aligned}
\operatorname{cum}\left(\mathrm{x}_{i}, \mathrm{x}_{j}^{T}, \mathrm{x}_{k}, \mathrm{x}_{l}^{T}\right)=\mathrm{E}\left[\mathrm{x}_{i} \mathrm{x}_{j}^{T} \mathrm{x}_{k} \mathrm{x}_{l}^{T}\right]-\mathrm{E}\left[\mathrm{x}_{i} \mathrm{x}_{j}^{T}\right] \mathrm{E}\left[\mathrm{x}_{k} \mathrm{x}_{l}^{T}\right] & \\
- & \mathrm{E}\left[\mathrm{x}_{i} \mathrm{x}_{k}\right] \mathrm{E}\left[\mathrm{x}_{j}^{T} \mathrm{x}_{l}^{T}\right] \\
& -\mathrm{E}\left[\mathrm{x}_{i} \mathrm{x}_{l}^{T}\right] \mathrm{E}\left[\mathrm{x}_{j}^{T} \mathrm{x}_{k}\right] 1 \leq i, j, k, l \leq M
\end{aligned}
$$

Where xi denotes $x_{i}(t)$ for short, $E[\cdot]$ denotes expectation, the superscript $T$ denotes complex conjugate. The Equation (3) is mapped into the Equation (4), according to the additivity and the multilinearity of the cumulants in the addition of independent variables, the expression as follows:

$$
\begin{aligned}
\operatorname{cum}\left(\mathrm{x}_{i}, \mathrm{x}_{j}^{T}, \mathrm{x}_{k}, \mathrm{x}_{l}^{T}\right) & =\sum_{p=1}^{N} \mathrm{a}_{i p} \mathrm{a}_{j p}^{T} \mathrm{a}_{k p} \mathrm{a}_{l p j}^{T T} \operatorname{cum}\left(\mathrm{s}_{p}, \mathrm{~s}_{p}^{T}, \mathrm{~s}_{p}, \mathrm{~s}_{p}^{T}\right) \\
& +\sum_{p=1}^{N} \operatorname{cum}\left(\mathrm{e}_{i}, \mathrm{e}_{j}^{T}, \mathrm{e}_{k}, \mathrm{e}_{l}^{T}\right) \quad 1 \leq i, j, k, l \leq M
\end{aligned}
$$

According to the Equation (5), a set of $\mathrm{M}^{4}$ fourth-order statistics can be obtained. In order to compute conveniently, the $\mathrm{M}^{4}$ matrix is mapped into $\mathrm{M}^{2} \times \mathrm{M}^{2} \mathrm{R}_{4}$ matrix as follows:

$\mathrm{R}_{4}((\mathrm{i}-1) \mathrm{M}+\mathrm{j},(\mathrm{k}-1) \mathrm{M}+\mathrm{l})=\operatorname{cum}\left(\mathrm{x}_{i}, \mathrm{x}_{j}^{T}, \mathrm{x}_{k}, \mathrm{x}_{l}^{T}\right)$

Then R4 can be the further simplified for the localization detection of the source, where $\otimes$ is Kronecker product [7]. 


$$
\begin{aligned}
\mathrm{R}_{4} & =\mathrm{E}\left\{(\mathrm{x} \otimes \mathrm{x})(\mathrm{x} \otimes \mathrm{x})^{H}\right\}-\mathrm{E}\{(\mathrm{x} \otimes \mathrm{x})\} \mathrm{E}\left\{(\mathrm{x} \otimes \mathrm{x})^{H}\right\}-\mathrm{E}\left\{\left(\mathrm{xx}^{H}\right)\right\} \otimes \mathrm{E}\left\{\left(\mathrm{xx}^{H}\right)\right\} \\
& =\mathbf{B}(r, \theta) \mathrm{C}_{s} B^{H}(r, \theta)+\mathbf{B}(r, \theta) \mathrm{C}_{e} B^{H}(r, \theta)
\end{aligned}
$$

Where

$$
\begin{aligned}
& \mathbf{B}(r, \theta)=\left[b\left(r_{1}, \theta_{1}\right) \quad b\left(r_{2}, \theta_{2}\right) \bullet \bullet b\left(r_{N}, \theta_{N}\right)\right]=\left[\mathrm{a}\left(r_{1}, \theta_{1}\right) \otimes \mathrm{a}\left(r_{1}, \theta_{1}\right) \bullet \bullet \mathrm{a}\left(r_{N}, \theta_{N}\right) \otimes \mathrm{a}\left(r_{N}, \theta_{N}\right)\right] \\
& C_{s}=E\left\{(\mathrm{~s} \otimes \mathrm{s})(\mathrm{s} \otimes \mathrm{s})^{H}\right\}-\mathrm{E}\{(\mathrm{s} \otimes \mathrm{s})\} \mathrm{E}\left\{(\mathrm{s} \otimes \mathrm{s})^{H}\right\}-E\left\{\left(\mathrm{ss}^{H}\right)\right\} \otimes \mathrm{E}\left\{\left(\mathrm{ss}^{H}\right)\right\} \\
& C_{s}=E\left\{(\mathrm{e} \otimes e)(\mathrm{e} \otimes e)^{H}\right\}-\mathrm{E}\{(\mathrm{e} \otimes e)\} \mathrm{E}\left\{(\mathrm{e} \otimes e)^{H}\right\}-E\left\{\left(\mathrm{ee}^{H}\right)\right\} \otimes \mathrm{E}\left\{\left(\mathrm{ee}^{H}\right)\right\}
\end{aligned}
$$

According to the MUSIC algorithm, if the signal are independent with each other, the matrix $\mathrm{R}_{4}$ can be decomposed into signal subspace and noise subspace, where the maximum eigenvector and eigenvalue correspond to the signal subspace $\mathbf{U}_{\mathbf{S}}$ and the others mean noise subspace $\mathbf{U}_{\mathbf{N}}$. Based on the orthogonal assumption between signal and noise, the angle and the distance detection of arrival can be estimated as follows, where $\|\bullet\|$ is matrix norm.

$$
\mathrm{P}(r, \theta)=\frac{1}{\left\|b^{\mathbf{H}}(r, \theta) \mathbf{U}_{N}\right\|^{2}}
$$

\section{Experiments}

Figure 3, show the rotor rubbing test table which is a rotary system containing three bearings and two cross components, these three bearings are sliding bearings which can simulate rotor imbalance, shaft system misalignment, rubbing and other faults. Rubbing of rotor and stator can be generated by the rubbing screw. The AE signal acquisition system made by PAC Corporation. Four UT-1000 sensors are arranged in a line at the end of the one side of arch waveguide plate. The coordinate origin is placed in the left end of the sensor array with $50 \mathrm{~mm}$ aperture. In the test, the rubbing screw rubbed the rotor at two the preset positions. The acquisition sampling frequency is set at $1 \mathrm{MHz}$, the duration time is $5 \mathrm{kms}$, the digital filter band is $1-200 \mathrm{KHz}$ and the pre-amplifier is $40 \mathrm{~dB}$. The preset rubbing positions are separately set at $\left(10 \mathrm{~mm}, 10^{\circ}\right)$ and $\left(120 \mathrm{~mm}, 60^{\circ}\right)$.

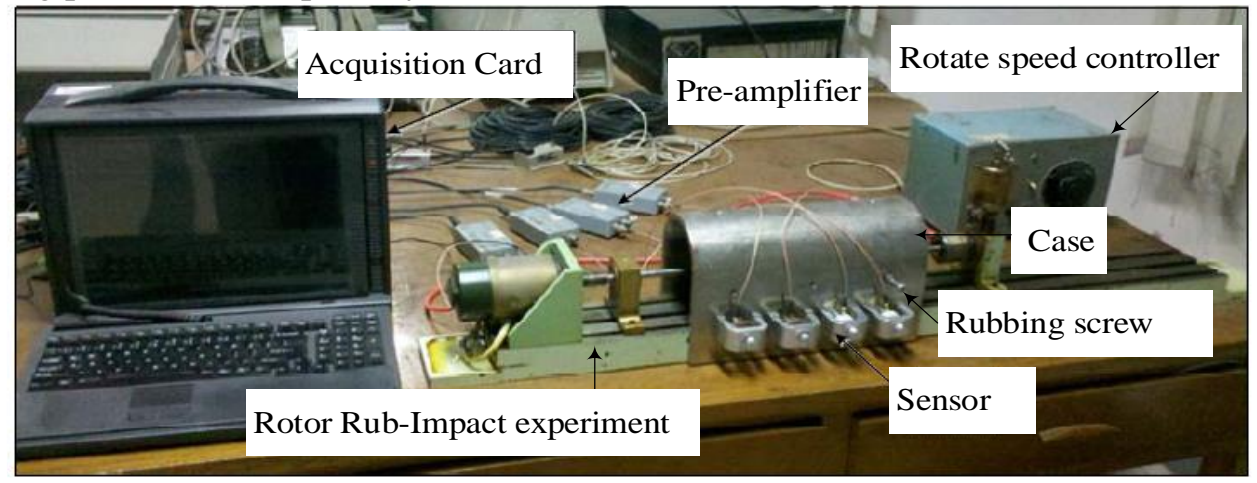

Figure 3. Experimental Setup 


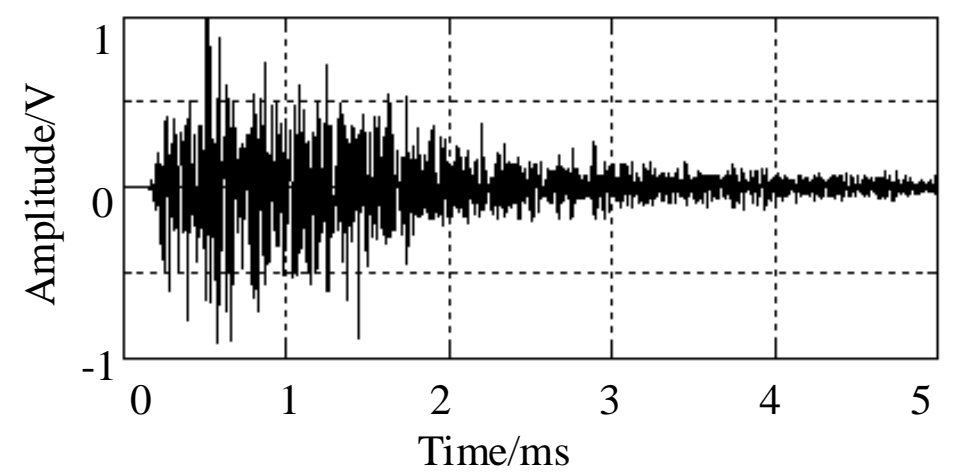

(a) Waveform in time domain

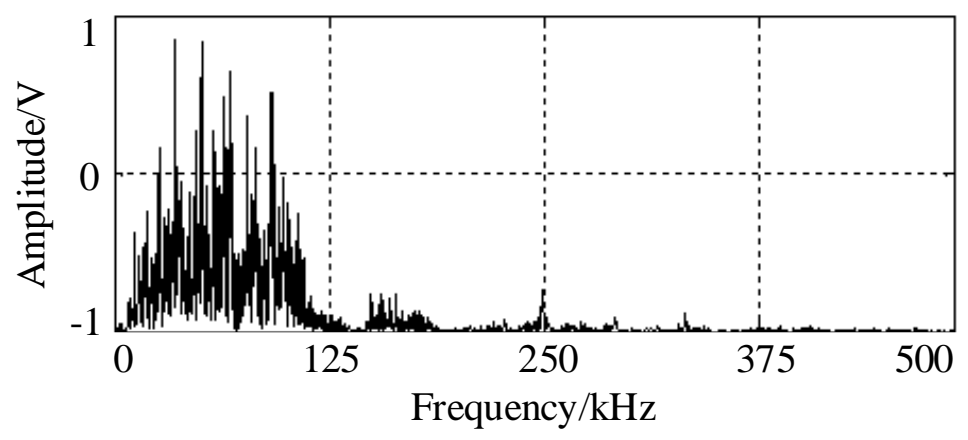

(b) Waveform in frequency domain

Figure 4. Rubbing AE Signal

Figure 4(a), shows the one $\mathrm{AE}$ event which records the entire two rubbing faults in time domain with 5120 points. Then the record is transformed into frequency domain using 8192 points FFT as shown in Figure 4(b). Due to the frequency dispersion effect the energy spread all over the frequency bins, but the major components are concentrated on the range from $0-100 \mathrm{KHz}$.

\section{Simulations and Evaluations}

\subsection{WPT for Zero-Order Modal Extraction}

Considering the AE propagation characteristics in steel plate with $10 \mathrm{~mm}$ thickness, the $\mathrm{S}_{0}$ and $\mathrm{A}_{0}$ modes served as the feature can be applied into the following localized algorithm. Figure 5, present the reconstructed AE record using Db11 wavelet 3 levels decomposition. However, combined with the previous researches, the initial rubbing flexural wave grows well [5]. Then the second envelop is extended by the interference from the first one. These two envelope peaks are obviously separated and approximates to the $\mathrm{A}_{0}$ from nearest rubbing fault and the ulterior fault. Figure 5(b), describes the feature in time-frequency (T-F) domain. 


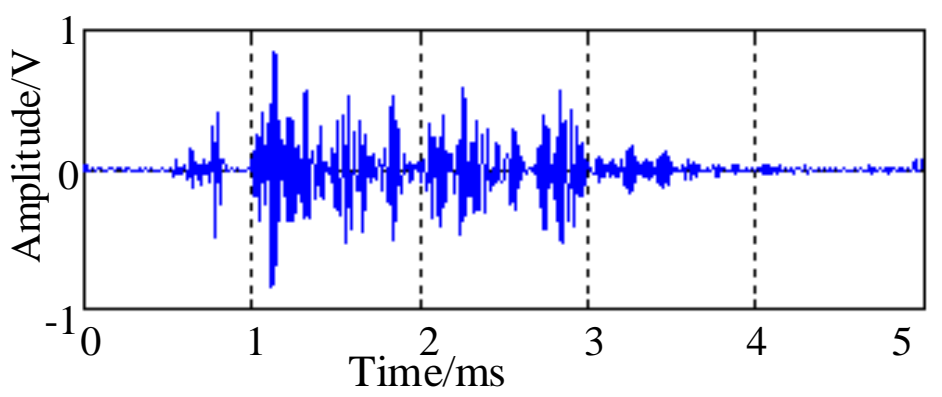

(a) Feature in time domain

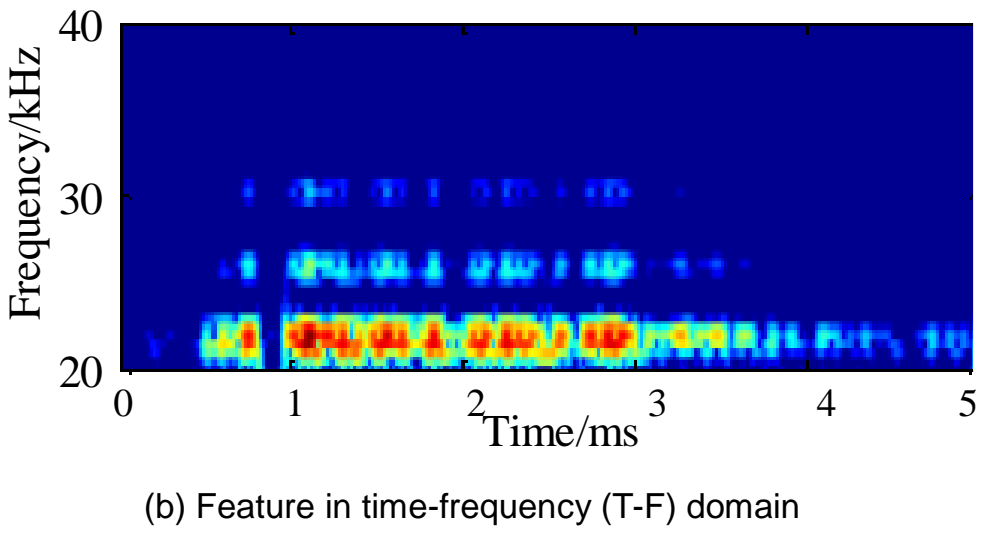

Figure 5. Reconstructed Each AE Event

\subsection{NFC-MUSIC Algorithm Localization Results}

Figure 6(a), presents the localization of the two simultaneous preset rubbing sources using NFC-MUSIC algorithm. The experiment result achieves the excellent localization performance. The spectrum peaks can be obviously shaped nearby the two rubbing position. Especially the near rubbing source can be detected sensitively and localized accurately. By contrast, the ulterior rubbing source without distinct sharp peaks achieves the lower localized accuracy due to the multipath interference from the first rubbing source.

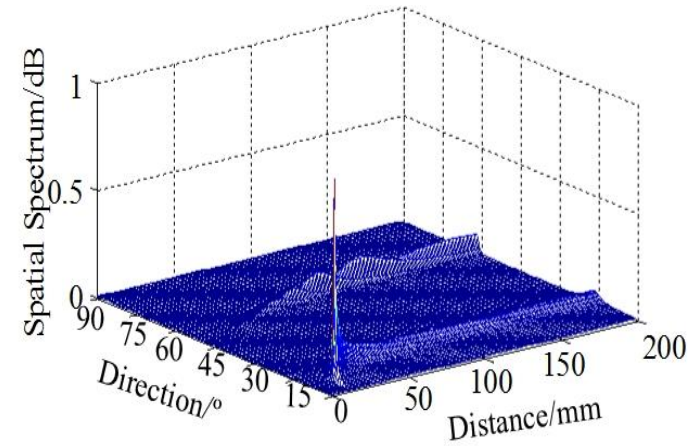

(a) The localization results of NFC-MUSIC 


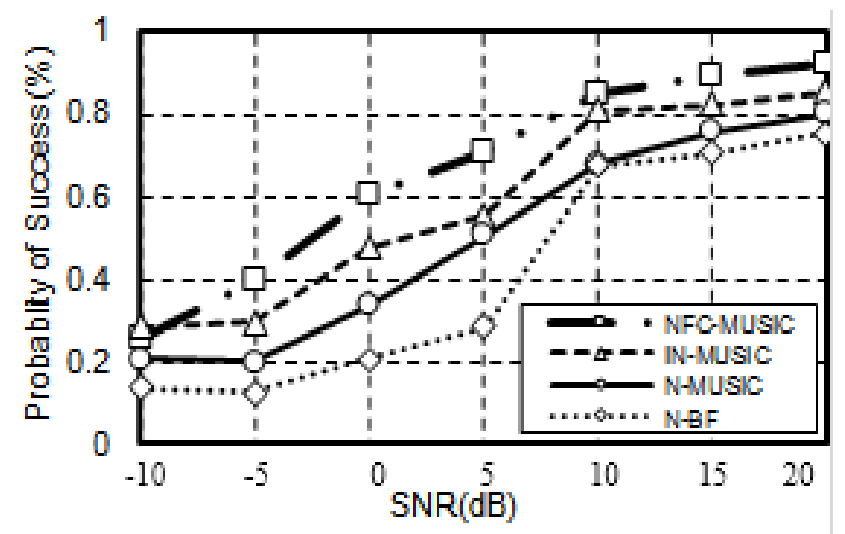

(b) The probability of success versus SNR

Figure 6. The Localization Result Analysis

Figure 6(b), presents the success probability comparison among the NFC-MUSIC, INMUSIC, N-MUSIC and N-BF algorithms. In this experiment, 400 Monto Carlo trials are performed to evaluate the probability of success the under different SNR. A trial is successful if it successes in distinguishing two source, i.e., the corresponding spatial spectrum has two peaks [9]. It is clearly shown that the NFC-MUSIC and IN-MUSIC obtain a large probability to localize double AE sources successfully than N-MUSIC and $\mathrm{N}-\mathrm{BF}$ method. It is because these front two algorithms can filer some coherent components away achieve the improved localization performance. Besides, the NFCMUSIC manifests the better anti-noise ability due to the increased virtual sensors. Therefore, NFC-MUSIC method can be applied to detect the rubbing sources in rotary machine for the incipient fault diagnosis.

\section{Conclusions}

This paper proposes a novel near-field multiple signal classification algorithm to detect multiple rubbing AE sources in rotor machinery. The superiorities of this algorithm to traditional methods are the feature narrow sub-band extracted using MPWT and WPT for overcoming dispersion effect and revised velocity

The superiorities of this algorithm to traditional methods are the extracted $\mathrm{S}_{0}$ and $\mathrm{A}_{0}$ through MPWT and WPT for overcoming dispersion effect and revised velocity, the NFCMUSIC algorithm for double AE sources decorrelation and increasing localized resolution. The experiment results show that NFC-MUSIC method can be served as the useful AE source localization methodology for the rotor rubbing fault diagnosis. Further work will continue to reduce the computation complexity for the workability of multi- rubbing sources detection on-line monitoring.

\section{Acknowledgements}

This work is supported by the National Nature Science Foundation of China (No.61231002, No.61375028). The authors thank all reviewers and editors, whose constructive comments help improve the presentation of this work.

\section{References}

[1] P. S. Keogh, "Contact dynamic phenomena in rotating machines: active/passive considerations", Mech. Syst. Signal Processing, vol. 29, no. 5, (2012), pp .19-33.

[2] M. Ahadi and M. S. Bakhtiar, "Leak detection in water-filled plastic pipes through the application of tuned wavelet transforms to acoustic emission signals", Applied Acoustics, vol. 71, no. 7, (2010), pp. 634-639.

[3] T. He, D. Xiao and Q. Pan, “Analysis on accuracy improvement of rotor-stator rubbing localization 
based on acoustic emission beamforming method", Ultrasonics, vol. 54, no. 1, (2014), pp. 318-329.

[4] A. Deng, H. Tong, J. Tang, "Study on Location Algorithms of Beamforming based on MVDR", Applied Mathematics \& Information Sciences, vol.7, no. 6, (2013), pp. 2455-2466.

[5] G. C. McLaskey, Steven D. Glaser and C. U. Grosse, "Beamforming array techniques for acoustic emission monitoring of large concrete structures", Journal of Sound and Vibration, vol. 329, no.12, (2010), pp. 2384-2394.

[6] J. Li, A. D. Deng, D. Y. Liu, R. Zhang and L. Zhao, "Near-Field Multiple Signal Classification Algorithm for Acoustic Emission Source Localization in Rolling Element Rub-Impact Fault Diagnostics", ICIC EXPRESS LETTERS, vol. 10, no. 3, (2016), pp. 663-668.

[7] Z. Yongteng, Y. Shenfang and L. Qiu, "Multiple fault detection on aircraft composite structures using near-field MUSIC algorithm", Sensors and Actuators A: Physical, vol. 214, (2014), pp. 234-244.

[8] J. L. F. Chacon, V. Kappatos and W. Balachandran, "A novel approach for incipient defect detection in rolling bearings using acoustic emission technique", Applied Acoustics, vol. 89, (2015), pp. 88-100.

[9] W. J. Zeng, X. L. Li and X. D. Zhang, "Direction-of-arrival estimation based on the joint diagonalization structure of multiple fourth-order cumulant matrices", Signal Processing Letters IEEE, vol. 16, no. 3, (2009), pp. 164-167.

[10] N. Yuen and B. Friedlander, "DOA estimation in multipath: an approach using fourth-order cumulants", Signal Processing IEEE Transactions on, vol. 45, no. 5, (1997), pp. 1253-1263.

\section{Authors}

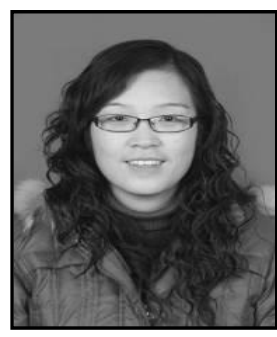

Jing Li received the B.A. degree (2005) from Hebei University of Engineering, the M.S. degrees (2008) from China University of Mining \& Technology. She is currently pursuing the Ph.D. degree at south-east University. Her main research interests include signal processing, sensor array technology and artificial intelligent, etc.as applied to AE signal recognition applications.

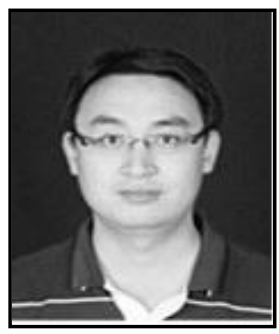

Yong Yang received his B.E degree (2004) from HeBei University of Engineering in 2004 and the M.S. Degree (2009) from China University of Mining and Technology. He is currently pursuing the Ph.D. degree at China University of Mining and Technology. His main research interests include signal processing, sensor array technology, and fault analyses as applied to AE signal recognition applications.

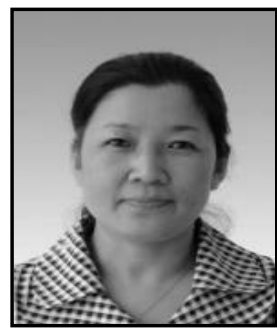

Li Xinghua She received her bachelor's degree (1990) and senior engineer (2001) in major Applied Physics. Now she is full senior researcher Chengdu BOE Optoelectronics Technology Co., Ltd. Her current research interests include TFT -LCD and AMOLED products.

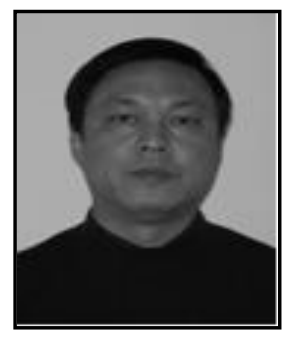

Li Zhao received his B.E.degree (1982) in automation from Nanjing University of aeronautics and astronautics, the M.S. degree (1988) from Southeast University and the Ph.D. degree (1995) from Kyoto Institute of Technology. Currently, he is a professor with department of radio engineering, Southeast University, Nanjing, China. He worked in areas of speech recognition, fault analyses and signal processing. Recently he has been working on acoustic emission technology for rotating machinery fault diag-nosis application. 
International Journal of Signal Processing, Image Processing and Pattern Recognition Vol. 9, No. 11, (2016) 